\title{
A new MEFV gene mutation in an Iranian patient with familial Mediterranean fever
}

\author{
S. Farjadian1, F. Bonatti ${ }^{2}$, A. Soriano ${ }^{3}$, M. Reina ${ }^{4}$, A. Adorni ${ }^{4}$, C. Graziano ${ }^{5}$, \\ M. Moghtaderi ${ }^{6}$, A. Percesepe ${ }^{2,4}$, G. Romeo ${ }^{5}$, D. Martorana ${ }^{4}$ \\ 'Department of Immunology, Shiraz University of Medical Sciences, Shiraz, Iran; \\ ${ }^{2}$ Department of Medicine and Surgery, University of Parma, Parma, Italy; ${ }^{3}$ Division of Rheumatology, \\ Arcispedale S. Maria Nuova di Reggio Emilia and Campus Bio-Medico University of Rome; \\ ${ }^{4}$ Unit of Medical Genetics, University Hospital of Parma, Italy; ${ }^{5}$ Medical Genetics Unit - S. Orsola Hospital, \\ University of Bologna, Italy; ${ }^{\circ}$ Allergy Clinic, Ali-Asghar Hospital, Shiraz University of Sciences, Shiraz, Iran
}

\begin{abstract}
SUMMARY
Familial mediterranean fever (FMF) is an inherited autoinflammatory disorder characterized by recurrent episodes of fever and painful inflammation involving the intra-abdominal organs, the lungs and the joints, which is highly prevalent in specific ethnic groups including the Iranians.

We report a 12-year-old boy from Iran, with a clinical history of recurrent fever.

Based on the suggestive clinical data, mutational analysis revealed the presence of the novel c.1945C $>\mathrm{T}$ heterozygous variant in exon 10, which leads to a leucine to phenylalanine change at position 649 of the protein. The mutation was inherited from the mother.

This novel mutation lies in exon 10 of the $M E F V$ gene, which encodes for a domain called B30.2-SPRY, located in the $\mathrm{C}$-terminal region of the pyrin protein and contains the most frequent mutations associated with FMF. The present report expands the spectrum of $M E F V$ gene mutations associated with FMF.

The uniqueness of this study, compared with other published case reports, consists in the new mutation found in the $M E F V$ gene. In fact, new mutations in this gene are of high interest, in order to better understand the role of this gene in autoinflammation.
\end{abstract}

Key words: Familial Mediterranean fever; MEFV gene; new mutation; autoinflammatory disorders; Pyrin protein.

\section{CASE REPORT}

Tamilial mediterranean fever (FMF) is 7 an inherited autoinflammatory disorder characterized by recurrent episodes of fever and serositis, which is highly prevalent in specific ethnic groups, including the Iranians (1). In patients affected by FMF, more than 300 mutations in the $M E F V$ gene, in particular in the exon 10 , have been described, demonstrating the great interest for the role of the $M E F V$ gene in the pathogenesis of FMF.

We report the case of a 12-year-old boy, son of non-consanguineous parents from southwestern Iran with a clinical history of recurrent episodes of fever beginning at the age of three, characterized by episodes of hyperpyrexia $\left(\max 41^{\circ} \mathrm{C}\right)$ every 3 to 5 weeks and lasting 3 to 5 days, associated with abdominal pain, nausea, vomiting and general myalgia, without arthritis or skin manifestations.

Both parents and the one-year-old sister were healthy with no history of periodic fever or other recurrent clinical manifestations.

His weight and height were in the normal range. The laboratory examinations during the attacks showed hemoglobin $13 \mathrm{~g} /$ $\mathrm{dL}$, white blood cell count (WBC) 10,000/ $\mu \mathrm{L}$ with $65.4 \%$ neutrophils, erythrocyte sedimentation rate (ESR) $40.8 \mathrm{~mm} / \mathrm{h}$ and C-reactive protein (CRP) $15.3 \mathrm{mg} / \mathrm{dL}$. The patient's ESR and WBC were normal in symptom-free periods. Liver function tests, electrolytes, blood urea nitrogen (BUN), creatinine and urine analysis were normal.
Corresponding author: Davide Martorana Unit of Medical Genetics University Hospital of Parma via Gramsci, 14 - 43126 Parma, Italy E-mail:dmartorana@ao.pr.it 
He showed negative tests for antinuclear antibodies (ANA) and rheumatoid factor, normal IgD levels, as well as negative investigations for infectious etiologies including Toxoplasma, Rubella, CMV, HSV and HIV.

An autoinflammatory etiology of the disorder was then suspected and a trial therapy with colchicine $0.03 \mathrm{mg} / \mathrm{Kg} /$ day was started, with a good response leading in two months to the disappearance of the attacks. Therapy was discontinued after 12 months, with no relapse of fever episodes during the following two years and normality in all the laboratory tests.

\section{DISCUSSION AND CONCLUSIONS}

The diagnosis of FMF was proposed in the patient based on Tel-Hashomer criteria (2): febrile episodes with abdominal pain (major criterion), favorable response to colchicine and age of onset $<20$ years (minor criteria), severe attacks requiring bed rest, transient inflammatory response with rising WBC and ESR (supportive criteria). A genetic test for the $M E F V$ gene was performed in the patient by Sanger sequencing the exons 1-10, which revealed the novel heterozygous c. $1945 \mathrm{C}>\mathrm{T}$ mutation in exon 10 , leading to a leucine to phenylalanine change at position 649 of the protein (Fig- ure 1). The mutation, which is not reported in Infevers (3), ClinVar or 1000 genomes, was inherited from the mother. It lies in the exon 10 of the $M E F V$ gene, which encodes for a domain called B30.2-SPRY, located in the C-terminal region of the pyrin protein; this domain is considered crucial for the modulation of the inflammasome activity, consistent with the fact that at least 90 different mutations identified as diseasecausing are located in that exon $(4,5)$. The mutation in position 649 may then alter the function of pyrin, consequently suppressing apoptosis and IL-1 $\beta$ activation.

Since FMF is inherited mainly as a recessive disease, there may be a second mutation transmitted by the father that was not identified; as a matter of fact, the absence of any mutation in the second allele has been shown in $20-25 \%$ of patients with the clinical picture of FMF and a positive response to colchicine therapy (2). Other possible pathogenic models include a dominant mutation with incomplete penetrance or the interaction of genetic and environmental causes (6).

The present report expands the spectrum of $M E F V$ gene mutations associated with the clinical phenotype of FMF.

Acknowledgements: this study was supported by the Fondazione Emma ed Ernesto Rulfo per la Genetica Medica (Italy).

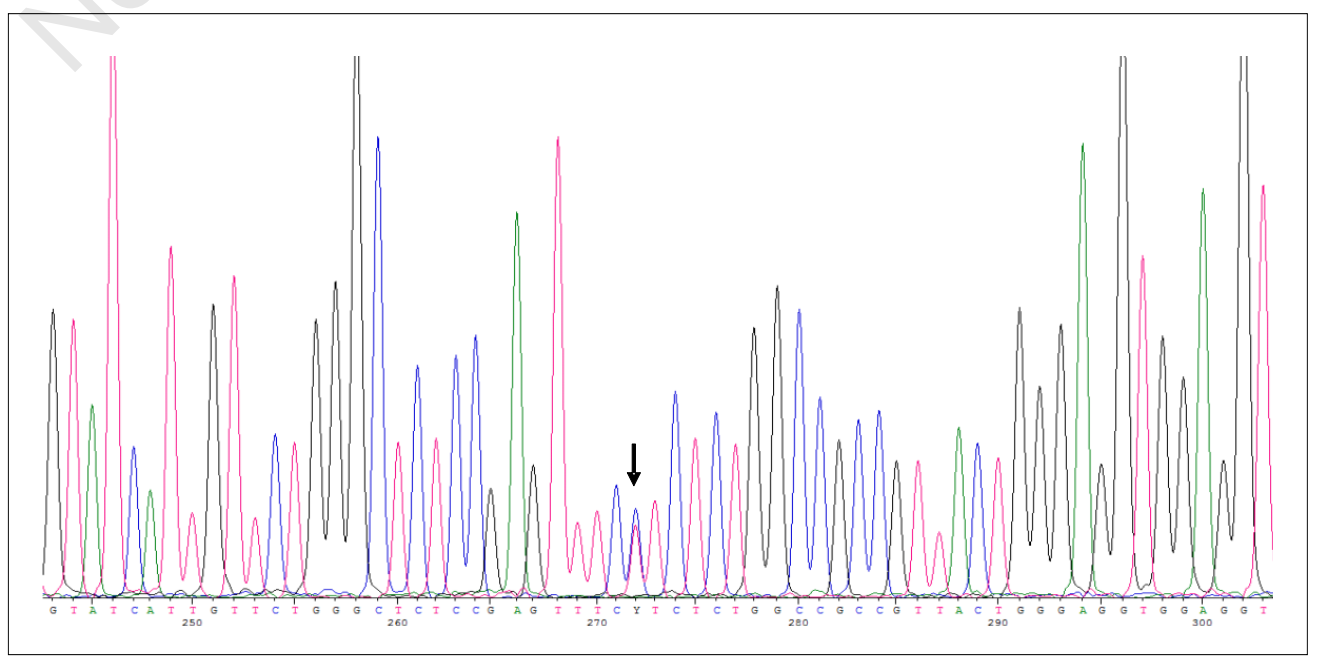

Figure 1 - Electropherogram of the novel c.1945C >T mutation in exon 10 in heterozygosity form, causing a leucine to phenylalanine change at position 649 of the protein (L649F). 


\section{REFERENCES}

1. Salehzadeh F. Familial Mediterranean fever in Iran: a report from FMF Registration Center. Int J Rheumatol. 2015; 912137.

2. Livneh A, Langevitz P, Zemer D, et al. Criteria for the diagnosis of familial Mediterranean fever. Arthritis Rheum. 1997; 40: 1879-85.

3. Infevers: the registry of hereditary auto-inflammatory disorders mutations. Available from: http://fmfighenrsfr/ISSAID/infevers

4. D'Cruz AA, Babon JJ, Norton RS, et al. Struc- ture and function of the SPRY/B30.2 domain proteins involved in innate immunity. Protein Sci. 2013; 22: 1-10.

5. Goulielmos GN, Fragouli E, Aksentijevich I, et al. Mutational analysis of the PRYSPRY domain of pyrin and implications for familial mediterranean fever (FMF). Biochem Biophys Res Commun. 2006; 345: 1326-32.

6. Booty MG, Chae JJ, Masters SL, et al. Familial Mediterranean fever with a single MEFV mutation: where is the second hit? Arthritis Rheum. 2009; 60: 1851-61. 